\title{
BREVE INTRODUCCIÓN A CORRIENTES POLIFÁSICAS
}

\section{BRIEF INTRODUCTION TO MULTIPHASE FLOWS}

Arturo Pérez París: Universidad de Alcalá de Henares. Madrid (España) arturo.perez@mixmail.com

\section{CURRÍCULUM VITAE}

Escuela Politécnica de la Universidad de Alcalá de Henares. Ingeniero electrónico y literato. Destacan sus estudios sobre aplicaciones eléctricas y motricidad, sobre los que ha publicado varios artículos científicos.

\section{RESUMEN}

El sistema polifásico de corrientes es el conjunto formado por varias corrientes alternas monofásicas, de igual frecuencia y valor eficaz, y que están desfasadas entre sí en un ángulo eléctrico igual a $360^{\circ}$ dividido por el número de fases. En el mundo industrial, los conceptos sobre corrientes polifásicas son muy importantes. Su estudio empieza por los conceptos de una sola corriente alterna, llamada también corriente alterna monofásica. En la práctica, resulta más interesante el uso simultáneo de varias corrientes monofásicas de iguales valores eficaces y frecuencia, pero de distinta fase, dando lugar así al sistema polifásico de corrientes alternas. De los sistemas bifásicos y los trifásicos hablaremos en este artículo. 


\section{PALABRAS CLAVE}

Sistema Polifásico - Sistema Bifásico - Sistema Trifásico - Corriente alterna

\section{ABSTRACT}

The current polyphase system is the set consisting of several single-phase alternating currents of equal frequency and rms, which are offset from each other in an electrical angle equals $360{ }^{\circ}$ divided by the number of phases. In the industrial world, concepts of polyphase currents are very important. His study begins with the concepts of alternating current one, also called single phase alternating current. In practice it is more interesting use of multiple single-phase currents and rms values equal frequency but different phase, thus leading to polyphase alternating current system. Of the three-phase two-phase systems and discuss in this article.

\section{KEY WORDS}

Polyphase system - Biphasic System - Three Phase System - Alternating Current

\section{ÍNDICE}

1. Introducción

2. Sistema bifásico

3. Sistemas trifásicos 


\section{TEXTO:}

\section{Introducción}

Alguien podría preguntarse qué hace un ingeniero de telecomunicaciones escribiendo sobre algo que es más propio de uno de industriales. La respuesta es bien sencilla. Actualmente me encuentro trabajando en este sector, por lo que es sencillo comprender que debo reciclar un poco mis conocimientos hacia este mundo. Así pues, además de aprender algo nuevo, me permito ponerlo de manifiesto aquí. Empecemos.

En el mundo industrial los conceptos sobre corrientes polifásicas son muy importantes. Su estudio empieza por los conceptos de una sola corriente alterna, llamada también corriente alterna monofásica. En la práctica, resulta más interesante el uso simultáneo de varias corrientes monofásicas de iguales valores eficaces y frecuencia, pero de distinta fase, dando lugar así al sistema polifásico de corrientes alternas.

En primer lugar, vamos a analizar algunos conceptos básicos. Por ejemplo, recibe el nombre de sistema polifásico de corrientes el conjunto formado por varias corrientes alternas monofásicas, de igual frecuencia y valor eficaz, y que están desfasadas entre sí en un ángulo eléctrico igual a $360^{\circ}$ dividido por el número de fases. Se denomina fase a cada una de las corrientes monofásicas que forman el sistema. Por excepción, recibe el nombre de sistema bifásico el sistema formado por dos fuerzas electromotrices alternas monofásicas desfasadas entre sí en $90^{\circ}$ y no en $180^{\circ}$ como se podía suponer atendiendo a la definición anterior.

Pese a que eléctricamente no hay limitación sobre el número de fases de un sistema polifásico, el más utilizado es el trifásico, por sus grandes ventajas: facilidad de 
transporte de la energía, extrema sencillez de algunas máquinas alimentadas por este sistema (motores asíncronos trifásicos), etc. También, aunque con mucha menos profusión, son utilizados los sistemas hexafásícos y dodecafásicos, sobre todo para la obtención de corrientes continuas rectificadas. La representación cartesiana de un sistema polifásico de corrientes consiste en un número de senoides igual al de fases del sistema, desfasadas un ángulo igual al característico del sistema.

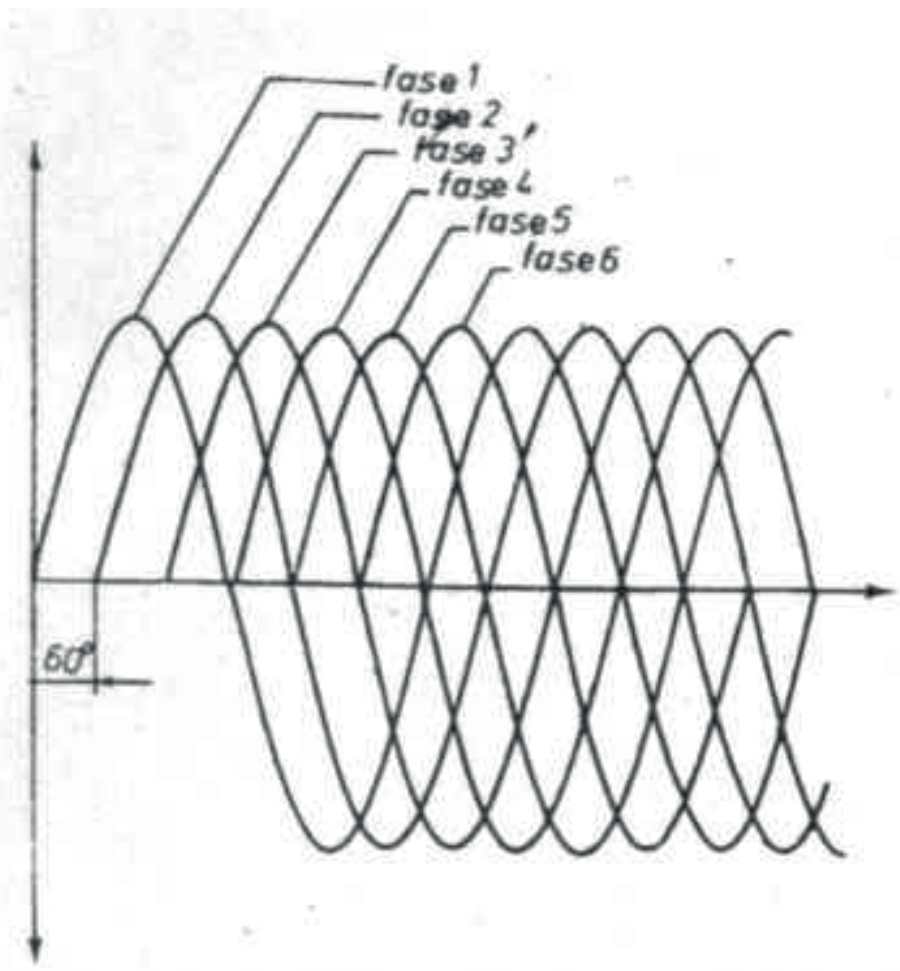

La representación vectorial se realiza trazando una estrella de tantos vectores iguales como fases tenga el sistema, formando cada dos vectores consecutivos un ángulo igual al característico del sistema, a saber: 


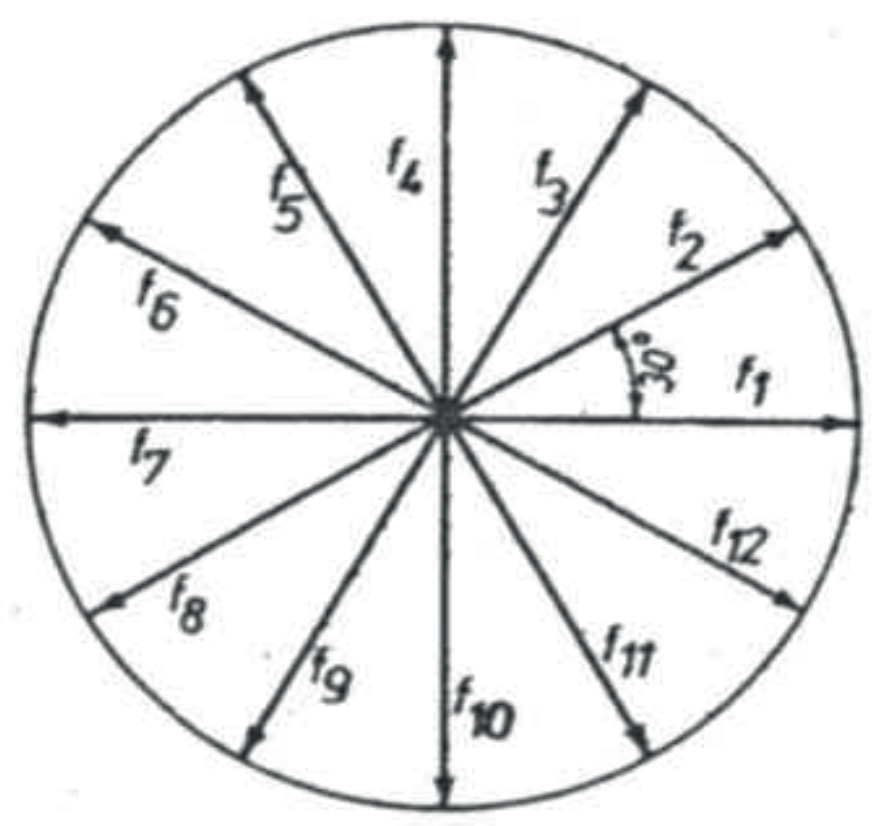

\section{Sistema bifásico}

Un ejemplo de la producción de energía polifásica, que nos ayudará a la comprensión de este tipo de corrientes, viene de la mano del alternador bifásico elemental. La rotación de dos bobinas perpendiculares entre sí en el interior de un campo magnético constante da lugar a la inducción de dos tensiones, una en cada bobina, desfasadas entre sí en $90^{\circ}$ eléctricos tal y como se muestra a continuación: 


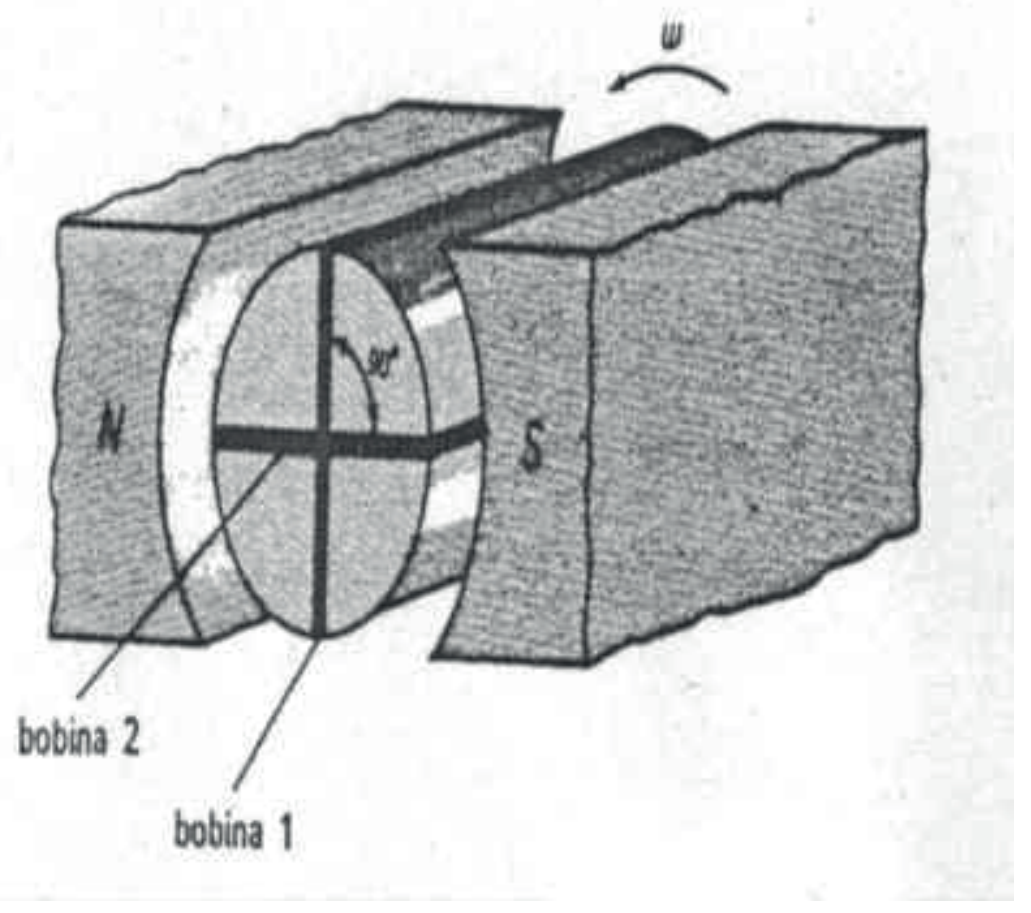

Obsérvese que cuando en la bobina 1 la inducción es máxima (valor máximo de fuerza electromotriz inducida), en la bobina 2 la inducción es mínima (valor cero de fuerza electromotriz inducida); sin embargo, cuando (las bobinas (rotor de la máquina)) hayan girado $90^{\circ}$, la situación será contraria a la anterior: en la bobina 1 la fuerza electromotriz inducida será nula y en la bobina 2 será máxima: 


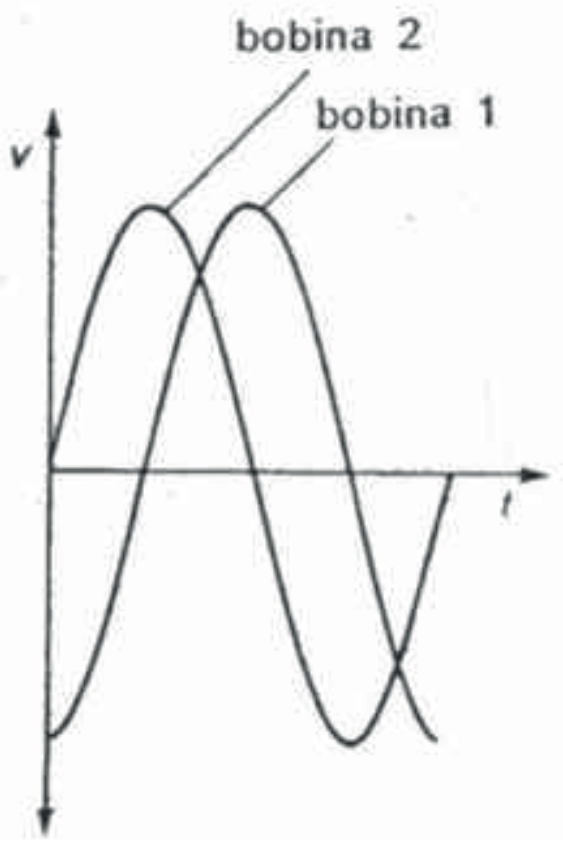

A continuación se representa, esquemáticamente, los bobinados del alternador elemental visto anteriormente, cuyos principios son $\mathrm{U}$ y V, y los finales X e Y. En la práctica, los finales $\mathrm{X}$ e Y se presentan unidos, quedando a la salida de la máquina sólo tres terminales: un hilo neutro (o común), que parte de la unión de los finales $\mathrm{Xe}$ Y, y dos hilos de línea, que parten de los principios U y V. Se verá ahora cómo están relacionadas las tensiones y corrientes en un sistema bifásico de tres hilos.

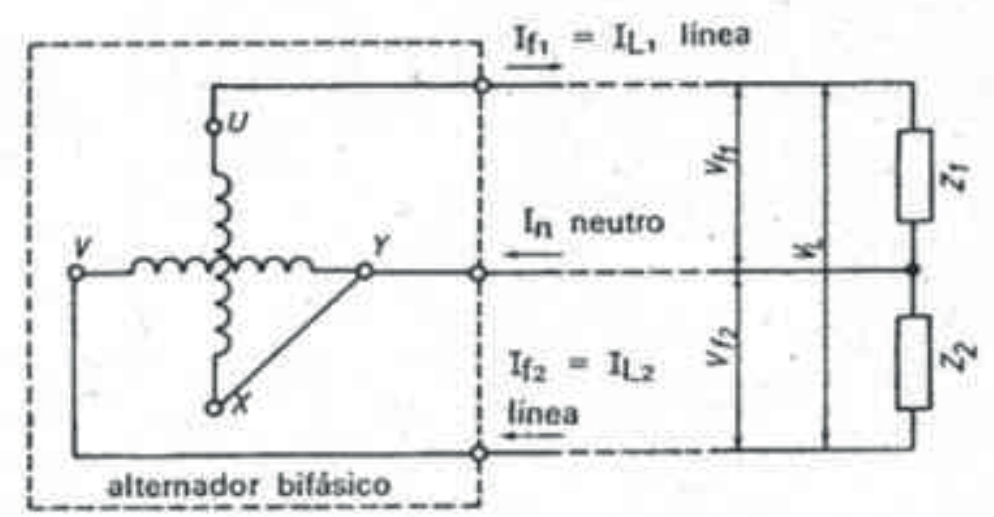


Al llegar a este punto, es necesario introducir dos definiciones válidas para todos los sistemas polifásicos:

- Se denomina tensión de línea o tensión compuesta.(V1) a la diferencia de potencial existente entre dos hilos de línea.

- Se denomina tensión de fase o tensión simple (Vf) a la diferencia de potencial existente entre un hilo de línea y el hilo neutro.

Para calcular la tensión compuesta existente en un sistema bifásico, bastará con hallar la suma geométrica de las tensiones producidas por cada una de las fases:

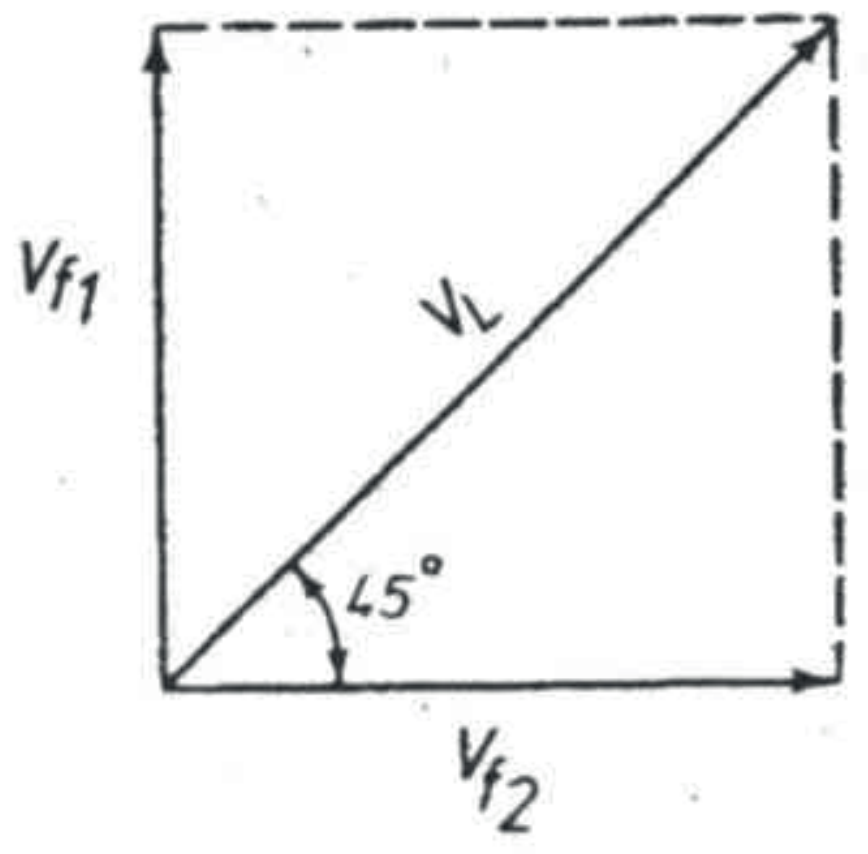

$V l=\sqrt{V f_{1}^{2}+V f_{2}^{2}}$

AI ser $\mathrm{Vf1}=\mathrm{Vf} 2=\mathrm{Vf}$, podemos escribir: 
$V l=\sqrt{2 \cdot V_{1}^{2}}=\sqrt{2} \cdot V f$

Esta expresión relaciona las tensiones simples y compuestas de un sistema bifásico. La intensidad que circula por el hilo neutro es también, en todo momento, la suma geométrica de las corrientes que circulan por los hilos de línea. Suponiendo igual carga (impedancia) para las dos fases, es decir $(\mathrm{Z1}=\mathrm{Z2})$, tendremos:

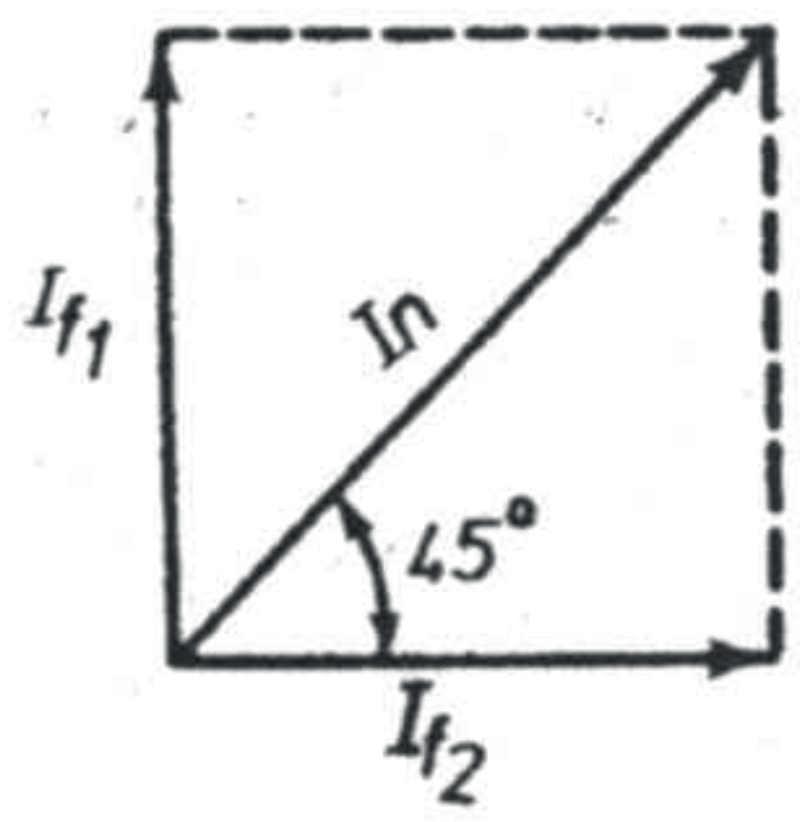

$I n=\sqrt{I f_{1}^{2}+I f_{2}^{2}}$

$\mathrm{Al}$ ser iguales las cargas, If 1 = If2 = If; por tanto:

$$
I n=\sqrt{2} I f
$$

Expresión que relaciona la corriente del neutro con la de línea. 
Así las cosas, siguiendo con la suposición de que el sistema es equilibrado (las cargas son iguales), la potencia activa total será dos veces la potencia activa disipada en una fase. Por tanto, siendo j el factor de potencia de la carga (ángulo de desfase entre tensión Vf y corriente If), tendremos:

\section{$P=2 \mathrm{Vf} \cdot$ If $\cdot \cos \varphi$}

Pero al ser:

$V f=\frac{V l}{\sqrt{2}} \quad$ y $\quad I f=\| l$

Podemos poner

$P=2 \cdot \frac{V l}{\sqrt{2}} \cdot h l \cdot \cos \varphi$

Y simplificando:

$P=\sqrt{2} \cdot V l \cdot n \cdot \cos \varphi$

Recordando las relaciones entre las potencias activa, reactiva y aparente, resulta:

$$
\begin{aligned}
& \mathrm{Pr}=2 \cdot \text { If } \cdot \text { If } \cdot \text { senw }=\sqrt{2} \cdot \mathrm{Vl} \cdot I l \cdot \text { sent } \\
& P a=2 \cdot V f \cdot I f=\sqrt{2} \cdot \mathrm{Vl} \cdot I l
\end{aligned}
$$




\section{Sistemas Trifásicos}

En estos momentos, después de ver este ejemplo conceptual de un sistema bifásico, estamos en condiciones de introducir los sistemas trifásicos de fuerzas electromotrices. La rotación de tres bobinas igualmente espaciadas en el interior de un campo magnético constante proporciona tres fuerzas electromotrices inducidas, desfasadas entre sí $120^{\circ}$ eléctricos:

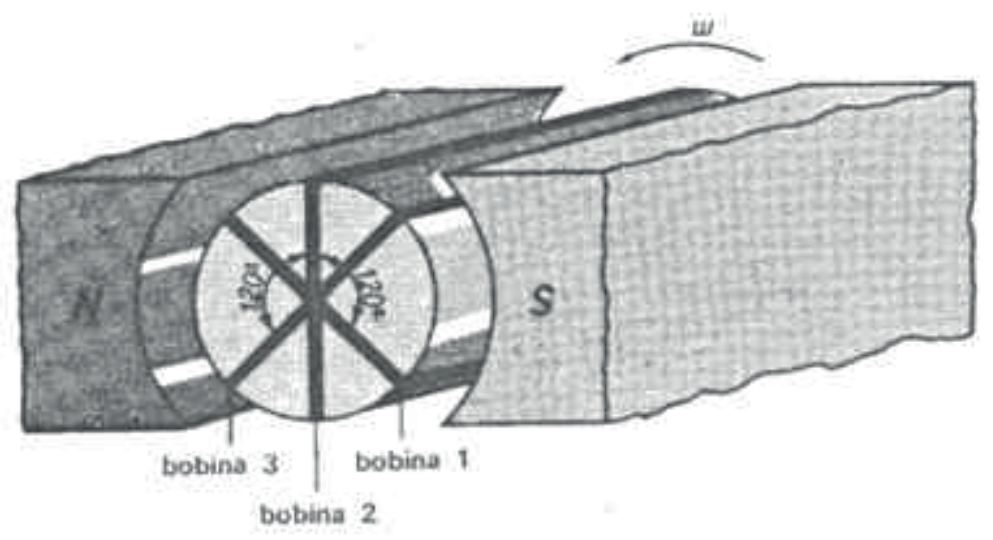

A continuación se representa el sistema de fuerza electromotriz que generaría el alternador trifásico elemental que acabamos de definir: 

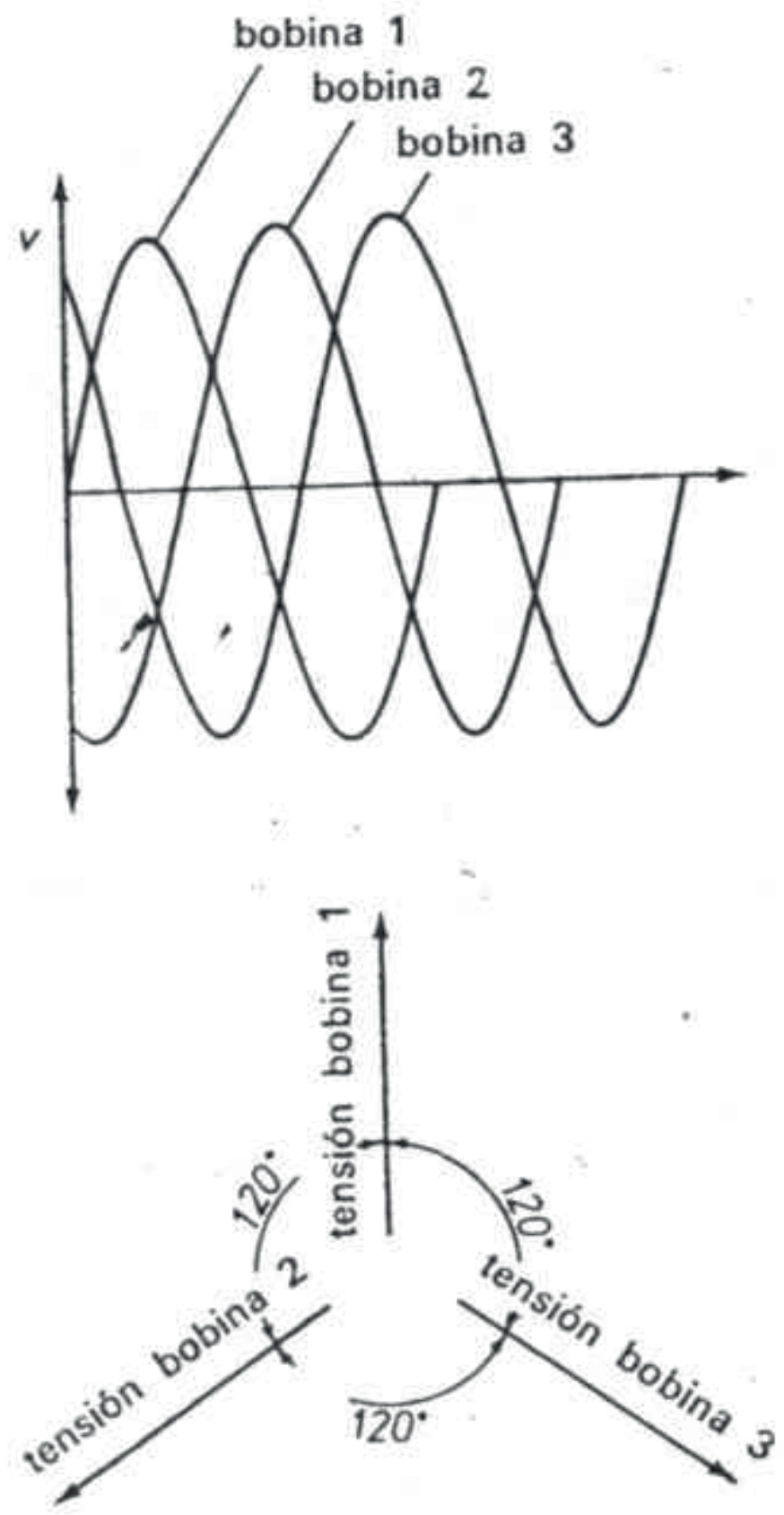

En la realidad los alternadores son muy distintos: el rotor es el inductor y está formado por uno o varios sistemas polares norte - sur, como se muestra a continuación: 

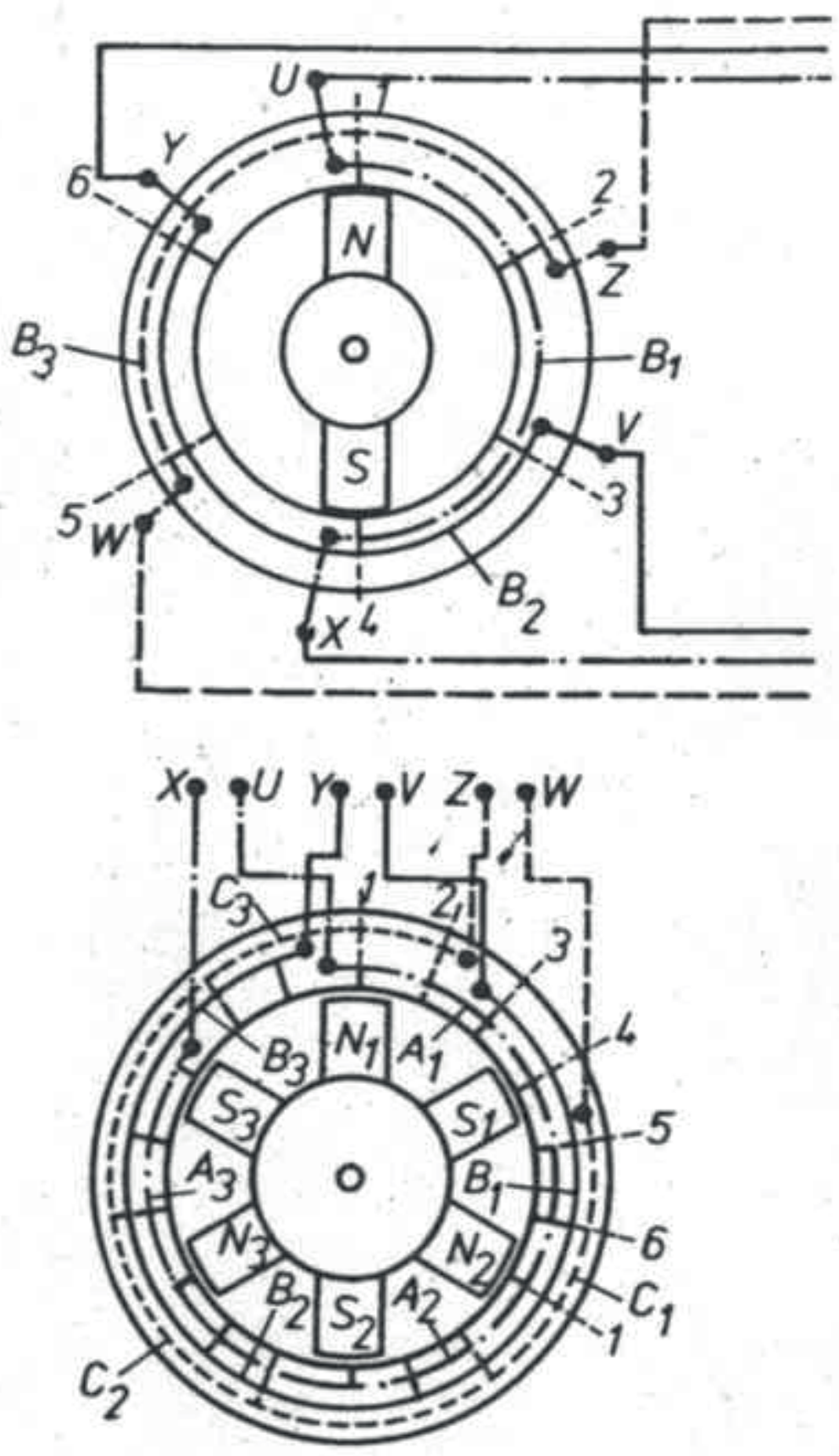

El estator (parte fija) es el inducido y está formado por tres paquetes de bobinas bien diferenciadas, cuyos principios se indican con las letras $\mathrm{U}, \mathrm{V}$ y $\mathrm{W}$, y sus finales, por $X, Y$ y $Z$ respectivamente. Los tres bobinados de un alternador trifásico pueden conectarse de dos modos distintos: conexión en estrella y conexión en triángulo; de forma que, a la salida de la máquina, sólo existen tres o, a lo sumo, cuatro terminales. 
La conexión del alternador en estrella (Y) consiste en unir los finales U V y W de las tres bobinas formando un punto común (denominado punto neutro) y dejando libres los tres principios $\mathrm{X}, \mathrm{Y}$ y Z. Del punto común suele sacarse un hilo conductor (denominado hilo neutro); que en ocasiones puede suprimirse y por el que en todo momento circula una corriente igual a la suma vectorial de las corrientes de fase. Seguidamente mostraremos las distintas posibilidades de tensiones de fase y línea en un alternador trifásico conectado en estrella. Obsérvese que entre ellas existen las siguientes relaciones:

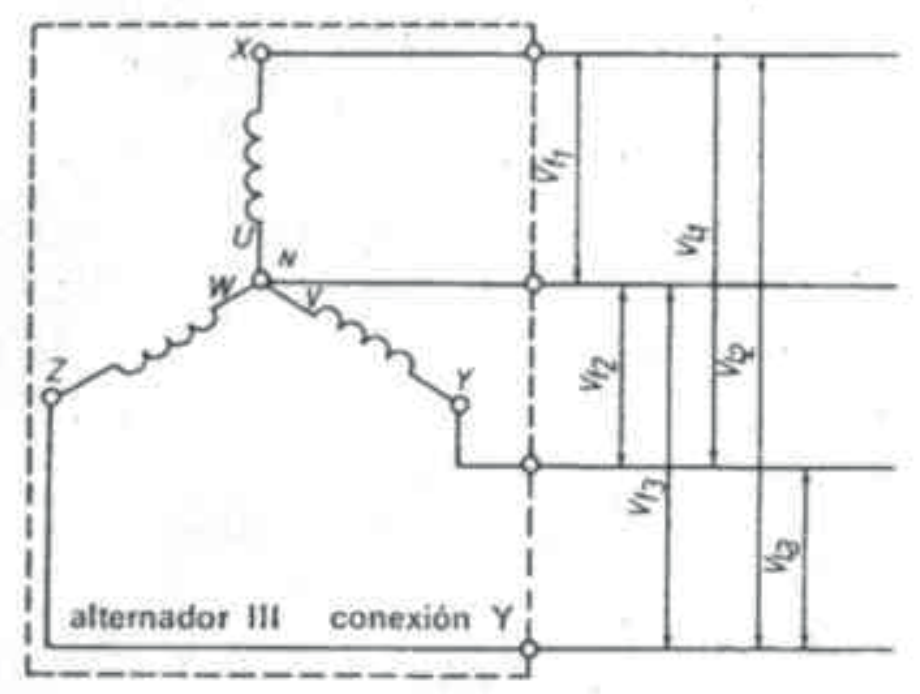

$$
\begin{aligned}
& \left|V l_{1}\right|=\left|V l_{2}\right|=\left|V l_{3}\right| \quad(a) \\
& V l_{1}+V l_{2}+V l_{3}=0 \quad \text { (b) } \\
& \left|V f_{1}\right|=\left|V f_{2}\right|=\left|V f_{3}\right| \quad(c) \\
& V f_{1}+V f_{2}+V f_{3}=0 \quad \text { (d) }
\end{aligned}
$$

Las igualdades (c) y (d) se deducen inmediatamente de la representación cartesiana o vectorial de la figura siguiente: 

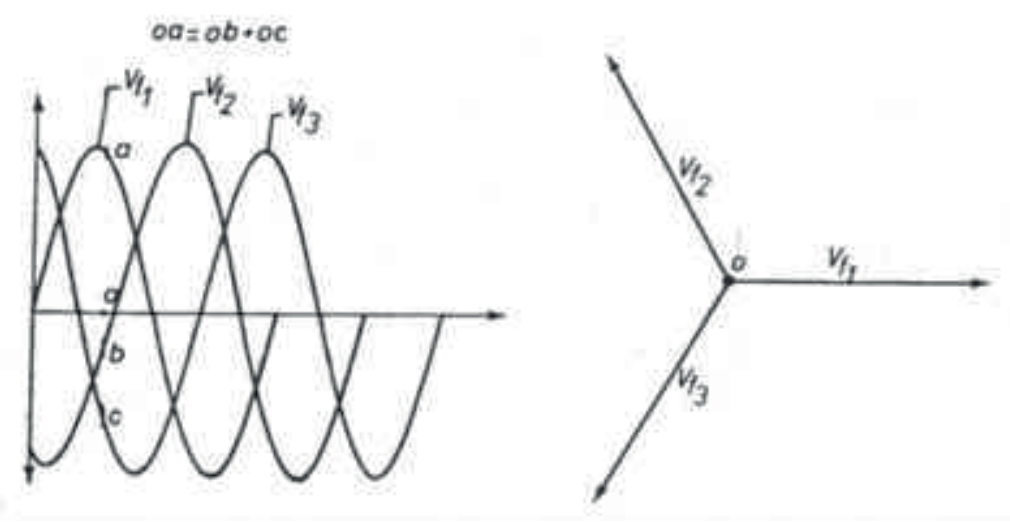

Las igualdades (a) y (b) se demostrarán en párrafos posteriores.

La conexión del alternador en triángulo (D) consiste en unir el principio de una fase con el final de la siguiente, repitiendo la operación cíclicamente hasta obtener un sistema cerrado.

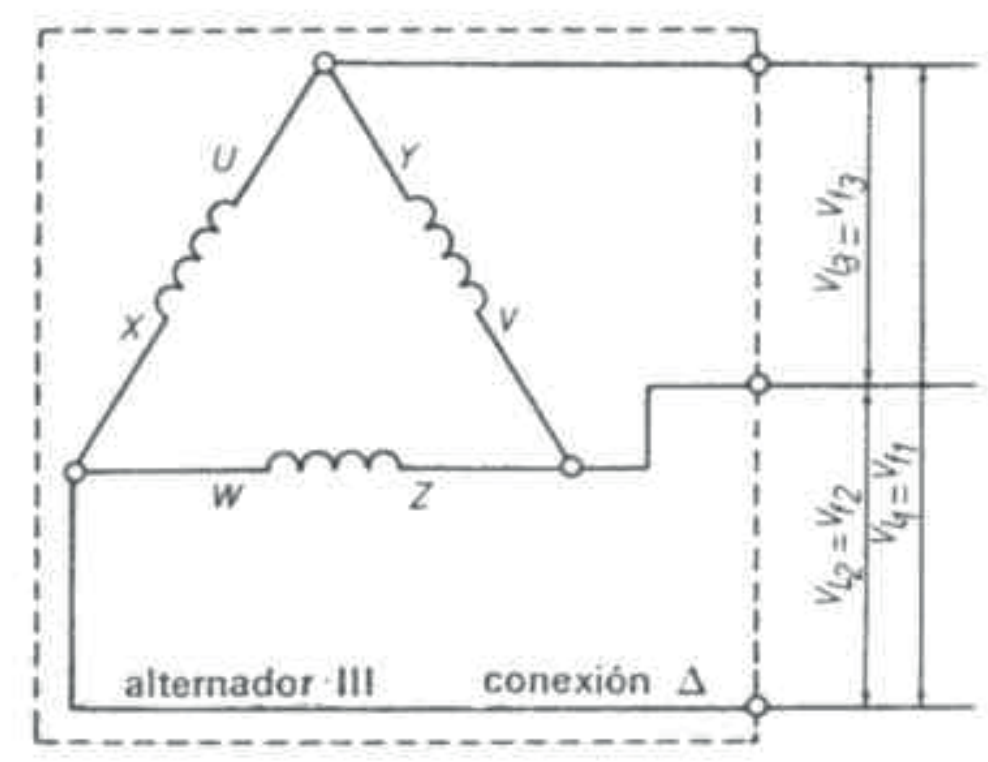

En este caso la tensión de fase es igual a la tensión de línea. Así, podemos verificar que: 
$\left|V l_{1}\right|=\left|V l_{2}\right|=\left|V l_{3}\right|$

Y, consecuentemente:

$V l_{1}+V l_{2}+\eta l_{3}=0$

Tensiones de un sistema trifásico en estrella con neutro

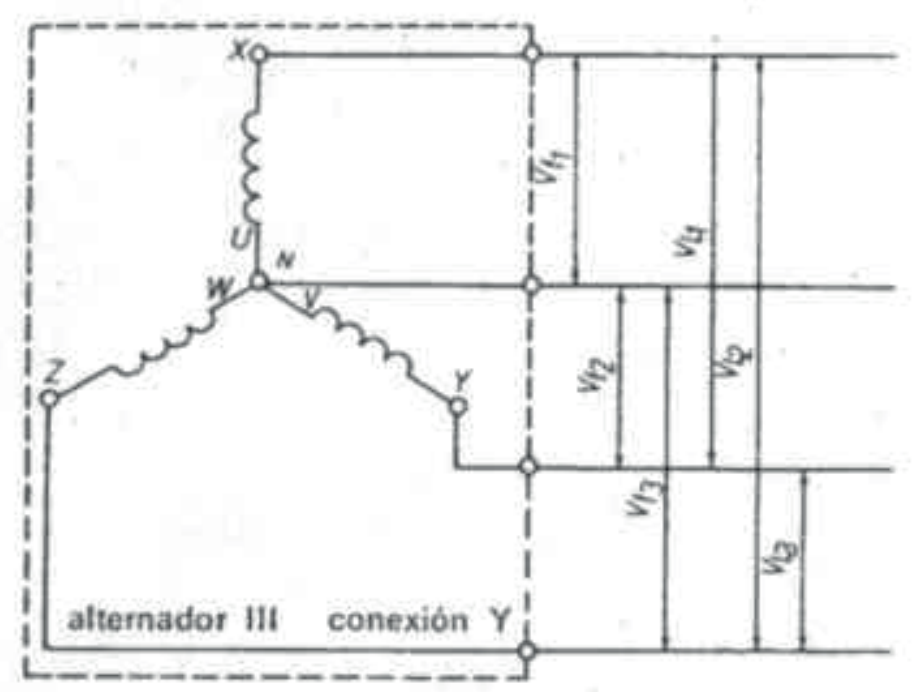

En este sistema en el que, al igual que en el bifásico a tres hilos, se dispone de dos tipos de tensiones (de fase y de línea), se efectúa el cálculo de éstas por el procedimiento de la suma vectorial en la representación de Fresnel y así, al observar la figura en la que se representa el alternador conectado en estrella, deducimos que:

$$
V_{X Y}^{*}=V_{X N}{ }^{*}+V_{N Y}^{*}=V_{X N}^{*}-V_{S N}^{*}
$$

Pero al ser: 
$\mathrm{VXY}=\mathrm{VL1}$

$\mathrm{VXN}=\mathrm{Vf1}$

$\mathrm{VNY}=\mathrm{Vf2}$

y haciendo:

$|V l|=\left|V l_{1}\right|=\left|V l_{2}\right|=\left|V l_{3}\right|$

$|V f|=\left|V f_{1}\right|=\left|V f_{2}\right|=\left|V f_{3}\right|$

Nos encontraremos con:

$\overline{V l 1}=\overrightarrow{V f 1}-\overrightarrow{V f 2}$

Y generalizando:

$\overrightarrow{V l}=\overrightarrow{V f}-\overrightarrow{V f}$

expresión que indica que la tensión de línea es igual a la diferencia geométrica de las tensiones de fase que la producen. El cálculo matemático del valor del módulo de la tensión en línea, en función de las tensiones de fase, es el siguiente: 


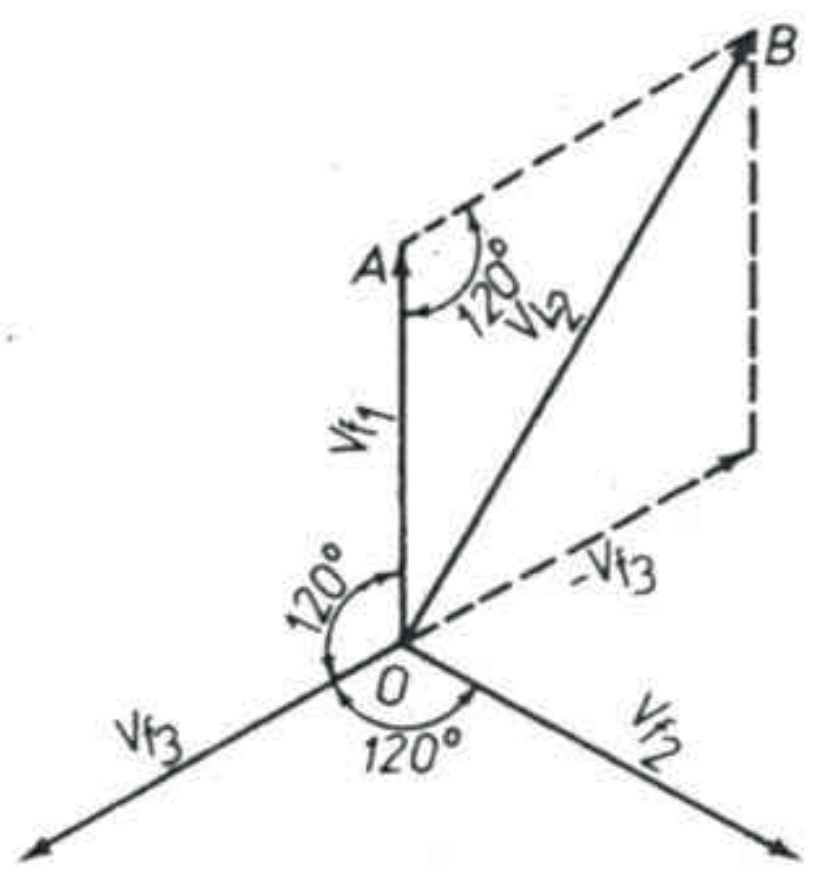

Aplicando el teorema del coseno al triángulo OAB obtenemos:

$V l=\sqrt{3} \cdot V f$

Prescindimos del desarrollo de los cálculos para llegar a este resultado, porque pueden encontrarse en cualquier libro de texto. Esta expresión liga la tensión de línea, o compuesta, con la tensión de fase, o simple, en un sistema trifásico. La representación de las tres tensiones simples y las tres compuestas puede hacerse también mediante el diagrama: 


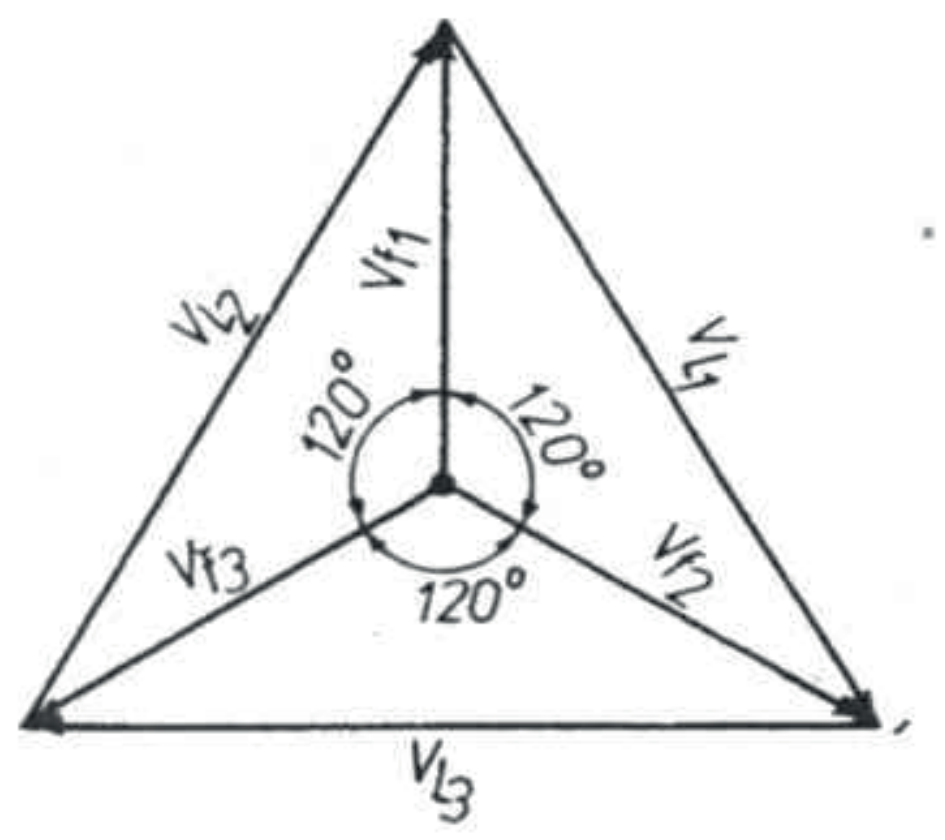

Evidentemente, en un sistema de distribución con alternador conectado en estrella, sin neutro, solamente existirán las tensiones de línea.

Tensión en un sistema trifásico en triángulo

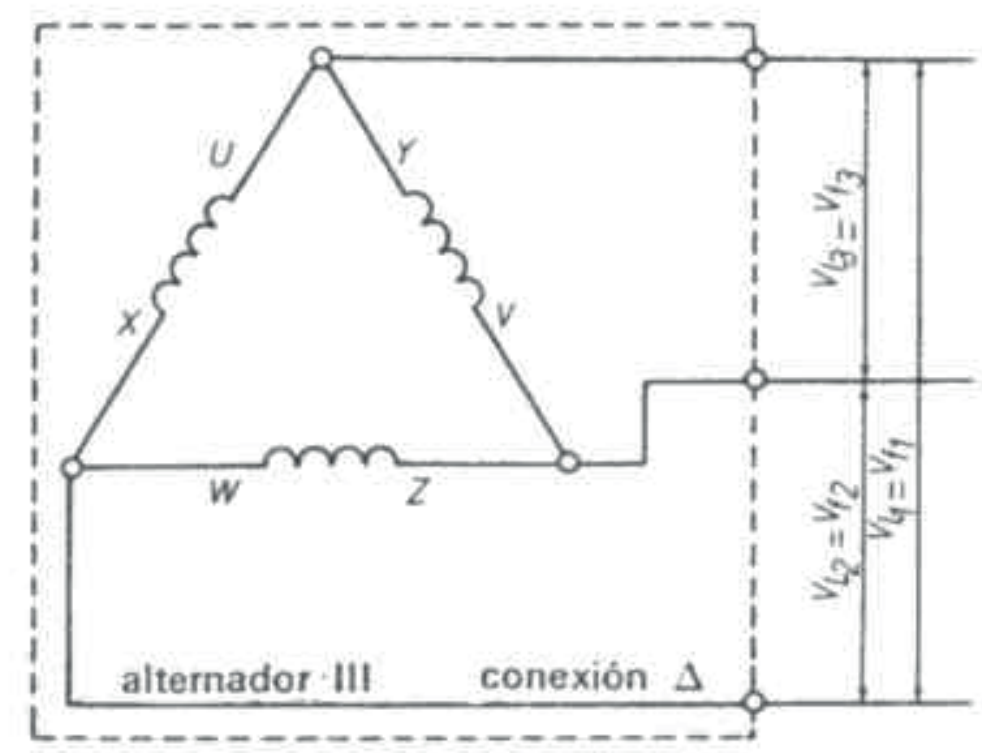


En este caso, como ya se ha dicho, la tensión de fase es igual a la de línea; por tanto:

$V l=V f$

Aunque la carga de los sistemas trifásicos se expondrá más adelante, téngase en cuenta que está constituida por tres impedancias que a su vez, podrán ir conectadas en estrella o en triángulo, según convenga: 

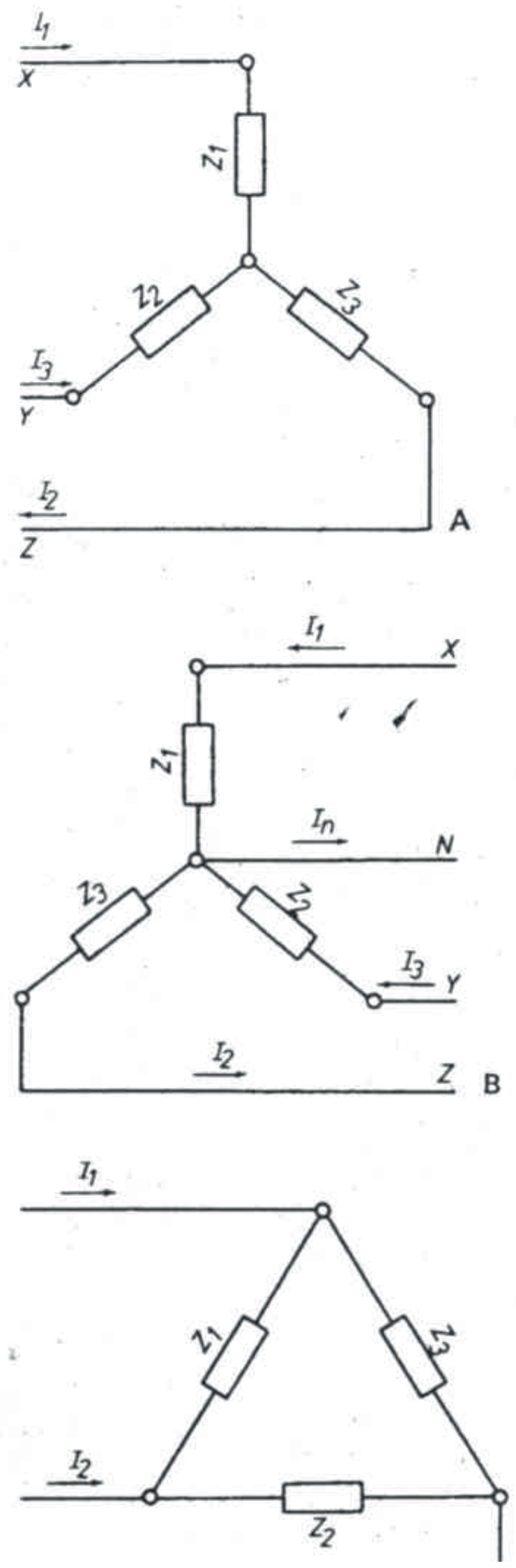
Nótese que, en el caso de conectar la carga en estrella, el neutro (punto común) podrá unirse al neutro del generador si éste tiene el hilo neutro, cosa que normalmente sucede, como es lógico pensar. Lógicamente, la naturaleza de la carga puede ser cualquiera: óhmica, óhmico-inductiva, óhmico-inductivo-capacitiva. Igualmente, las impedancias de la carga pueden ser iguales o distintas, dando lugar a sistemas trifásicos equilibrados o desequilibrados respectivamente. En la práctica las instalaciones trifásicas suelen ser desequilibradas.

Recuérdense dos definiciones válidas para todos los sistemas polifásicos:

-Se denomina corriente de fase aquella que recorre una fase de un sistema polifásico.

-Se define corriente de línea aquella que, saliendo de los bornes principales de un alternador, entra en los bornes de una carga.

Considérese el siguiente alternador trifásico, cuyos bobinados están conectados en estrella, que alimenta una carga trifásica conectada en triángulo. Según las definiciones anteriores, I1, I2 e I3 serán las corrientes de línea, las corrientes de fase en la carga serán If1, If2 e If3; y por último Ia, Ib e Ic son las corrientes de fase del alternador. 


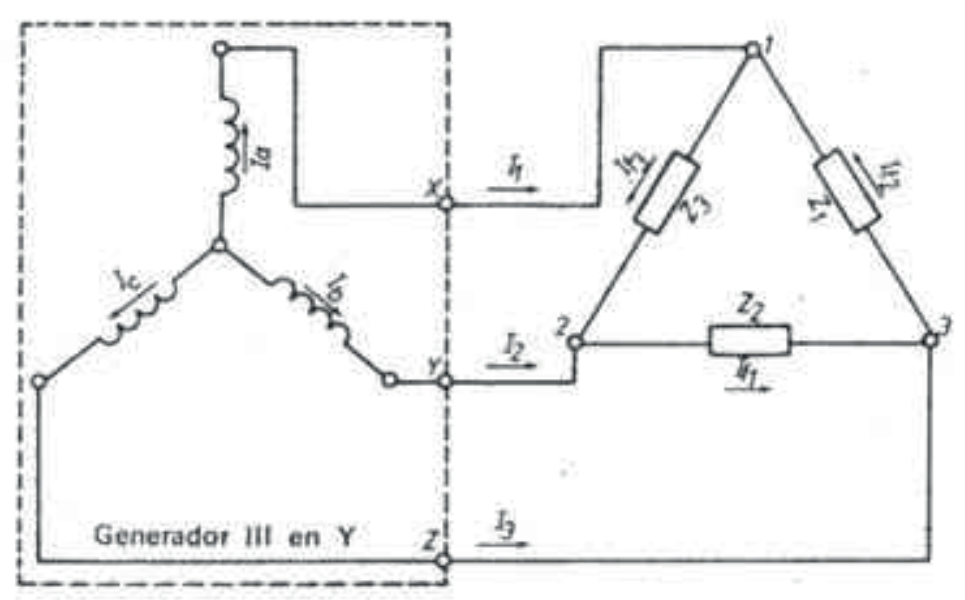

Obsérvese que, en un alternador conectado en estrella, las corrientes de línea coinciden con las corrientes de fase. En la carga del alternador (conectada en triángulo), fácilmente se podemos comprender que la corriente de cada una de las fases es distinta de las corrientes de línea. Para el cálculo de las corrientes de línea en función de las corrientes de fase en una carga conectada en triángulo, vamos a proceder de la siguiente forma:

Asígnese un sentido arbitrario a cada una de las corrientes de línea y fase, como lo hicimos en la anterior representación. Aplicando la primera ley de Kirchhoff a los nudos 1, 2 y 3, obtenemos un sistema de tres ecuaciones, fácilmente resoluble.

Nudo 1 :

$$
I_{1}+I_{12}=I_{13} \Rightarrow I_{1}=I_{13}-I_{12}
$$

Nudo 2:

$$
I_{2}+I_{13}=I_{11} \Rightarrow I_{2}=I_{11}-I_{13}
$$

Nudo 3:

$$
I_{3}+I_{11}=I_{12} \Rightarrow I_{3}=I_{12}-I_{11}
$$


Por otro lado si dicho sistema de ecuaciones es conjuntado, es decir, sumamos término a término las mismas obtenemos:

$\mathrm{I} 1+\mathrm{I} 2+\mathrm{I} 3=0$

De donde se deduce así la condición fundamental para las corrientes de línea cuando el sistema es de tres hilos: en todo momento, la suma vectorial de las corrientes de línea debe ser nula. Por tanto, las tres corrientes podrán ser representadas por un triángulo cerrado:

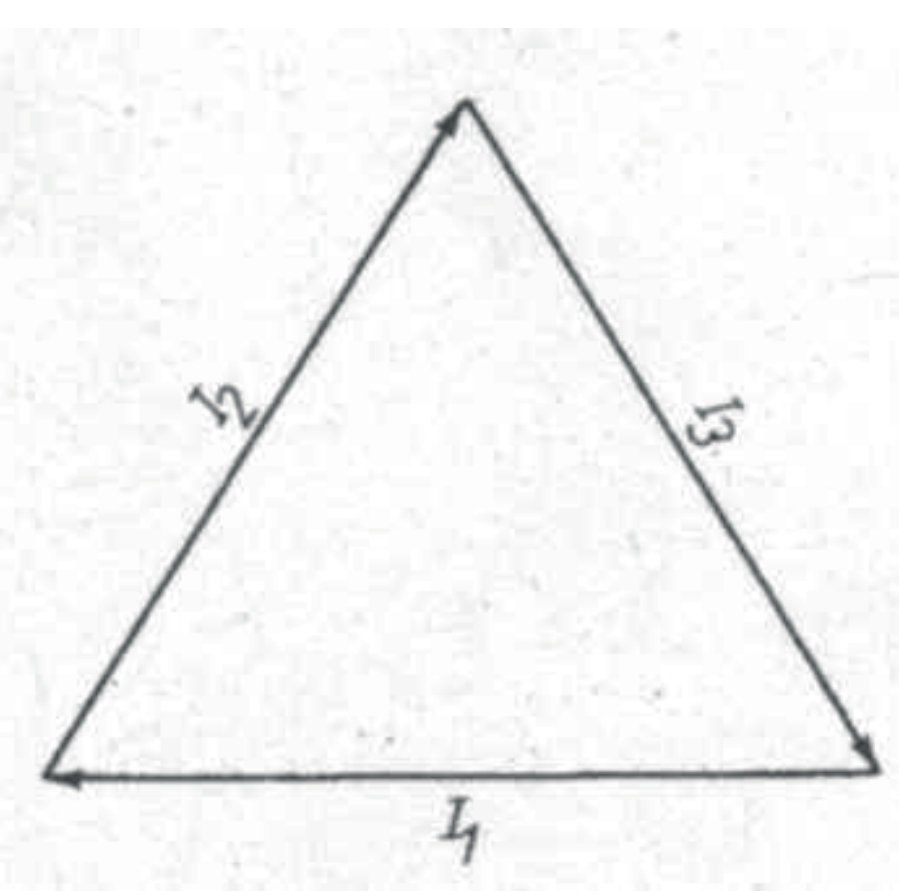

Quede como conclusión de ello, que las tres corrientes de fase se pueden representar por una terna de vectores (en estrella) procedentes de un punto común (neutro), y cuyos extremos se apoyan en los vértices del triángulo que representa las corrientes de línea. Si la carga es equilibrada, el centro de la estrella coincidirá con el baricentro del triángulo: 


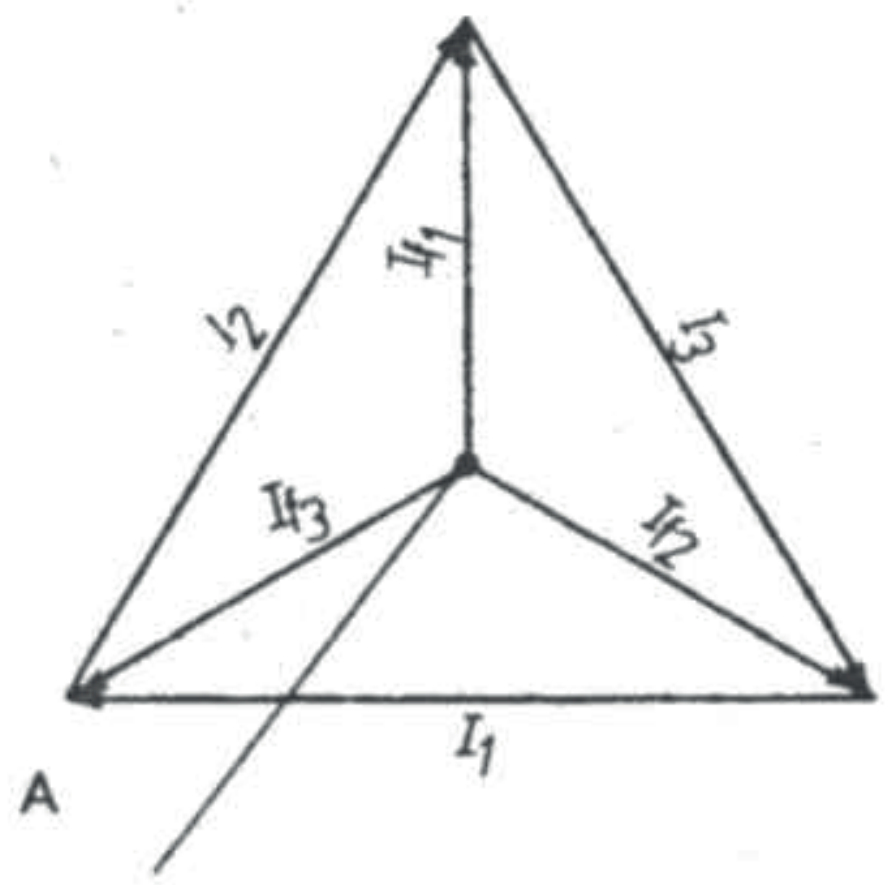

\section{baricentro}

en caso contrario, el centro de la estrella no coincidirá con el baricentro, es decir:

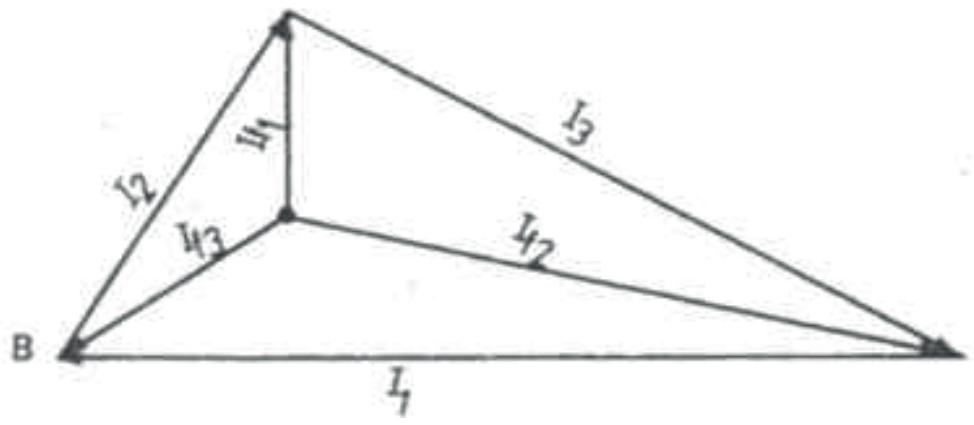

Obsérvese que cualquiera de los dos triángulos representados pueden deducirse de las ecuaciones de los nudos sugeridas anteriormente. 
Los sistemas trifásicos, debido a sus peculiaridades de conexión, tienen procesos de cálculo semejantes pero no iguales, como se verá a continuación. Supongamos una carga equilibrada en conexión estrella $(\mathrm{Y})$. Considérese también un sistema trifásico simétrico, que alimenta a la carga antes aludida formada por tres impedancias iguales $\mathrm{Z1}=\mathrm{Z2}=\mathrm{Z3}=\mathrm{Z}=\mathrm{R}+\mathrm{j} \mathrm{X}$ conectadas en estrella:

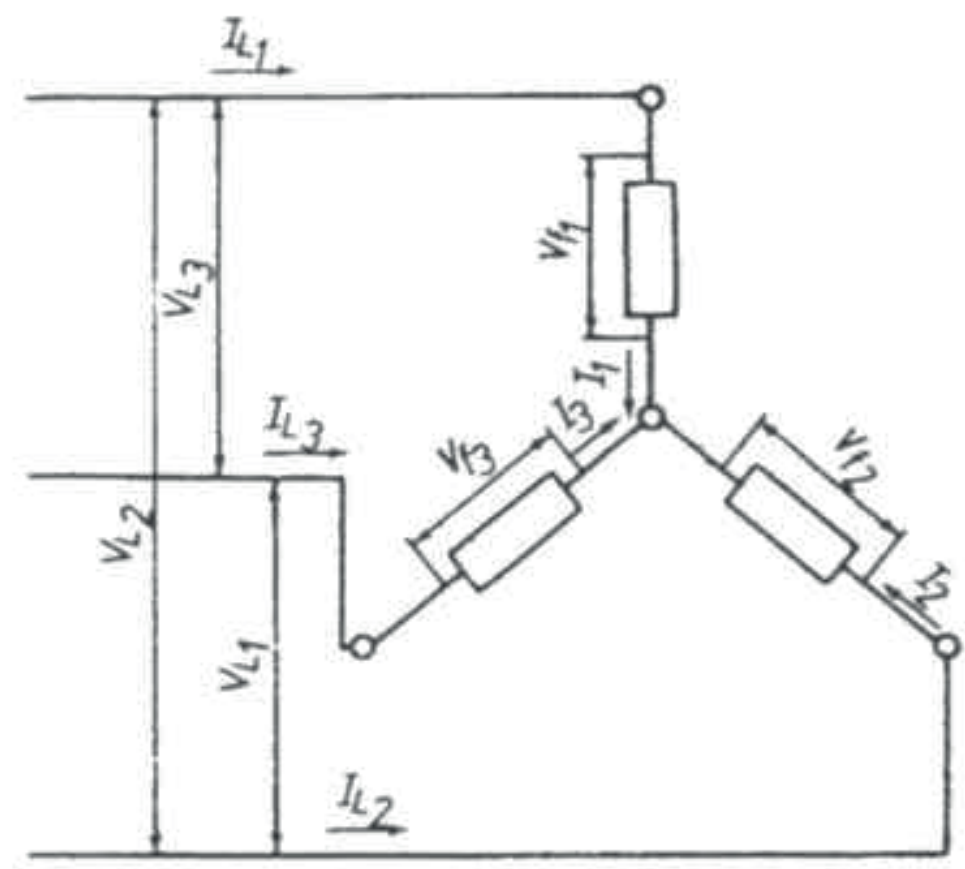

Las tensiones, en cada una de las fases, serán (recuérdese el carácter vectorial de tensiones, corrientes e impedancias):

$$
\begin{aligned}
& V f_{1}=Z 1 \cdot I_{1}=Z \cdot I_{1} \\
& V f_{2}=Z 2 \cdot I_{2}=Z \cdot I_{2} \\
& V f_{3}=Z 3 \cdot I_{3}=Z \cdot I_{3}
\end{aligned}
$$

Sumando miembro a miembro las tres igualdades, obtendremos: 
$\mathrm{Vf1}+\mathrm{Vf} 2+\mathrm{Vf} 3=\mathrm{Z} \cdot(\mathrm{I} 1+\mathrm{I} 2+\mathrm{I} 3)$

Recordando que:

$\mathrm{I} 1+\mathrm{I} 2+\mathrm{I} 3=0$

y al ser la impedancia propuesta no nula, necesariamente:

$\mathrm{Vf1}+\mathrm{Vf} 2+\mathrm{Vf} 3=0$

Esta ecuación demuestra que, al tener una carga formada por tres impedancias iguales conectadas en estrella, los vectores representativos de las tensiones de fase tienen resultante nula. Este es el motivo por el cual la terna de vectores saldrá del baricentro del triángulo de tensiones de línea:

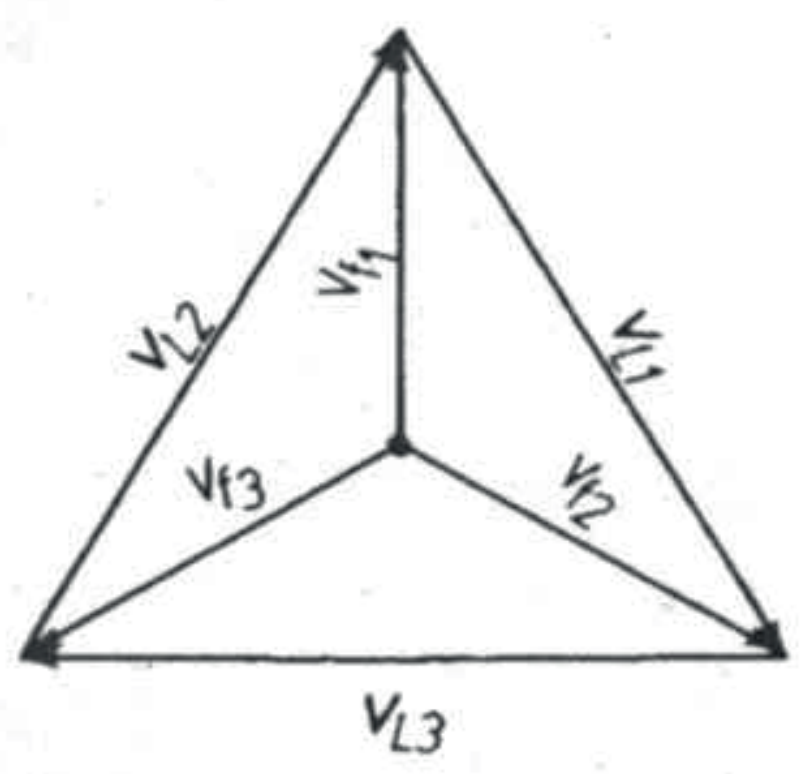


Asimismo, del sistema de tres ecuaciones anterior y por simple transposición de términos, obtendremos:

$I_{1}=\frac{V f_{1}}{Z} \quad I_{2}=\frac{V f_{2}}{Z} \quad I_{3}=\frac{V f_{3}}{Z}$

Y al ser

$$
\begin{aligned}
\left|I_{L 1}\right| & =\left|I_{f 1}\right|=\left|I_{1}\right| \\
\left|I_{L 2}\right| & =\left|I_{f 2}\right|=\left|I_{2}\right| \\
\left|I_{L 3}\right| & =\left|I_{f 3}\right|=\left|I_{3}\right| \\
\left|V_{L 1}\right| & =\left|V_{L 2}\right|=\left|V_{L 3}\right|=\left|V_{L}\right| \\
\left|V_{f 1}\right| & =\left|V_{f 2}\right|=\left|V_{f 3}\right|=\left|V_{f}\right|=\frac{\left|V_{L}\right|}{\sqrt{3}}
\end{aligned}
$$

Se puede poner 


$$
\begin{aligned}
& I_{1}=\frac{V_{f 1}}{Z}=L_{L}=\frac{V_{f}}{Z}=\frac{V_{L}}{\sqrt{3} Z} \\
& I_{2}=\frac{V_{f 2}}{Z}=I_{L}=\frac{V_{f}}{Z}=\frac{V_{L}}{\sqrt{3} Z}
\end{aligned}
$$

$$
I_{3}=\frac{V_{f 3}}{Z}=L_{L}=\frac{V_{f}}{Z}=\frac{V_{L}}{\sqrt{3} Z}
$$

Expresiones que indican que el valor de la corriente de fase de un sistema trifásico en estrella es igual al valor de la corriente de línea e igual, a su vez, a la tensión de línea dividida por veces la impedancia de una rama cualquiera. El diagrama vectorial de tensiones y corrientes podría ser el representado seguidamente, en el que se ha supuesto la carga óhmico-inductiva $Z=R+j X$.

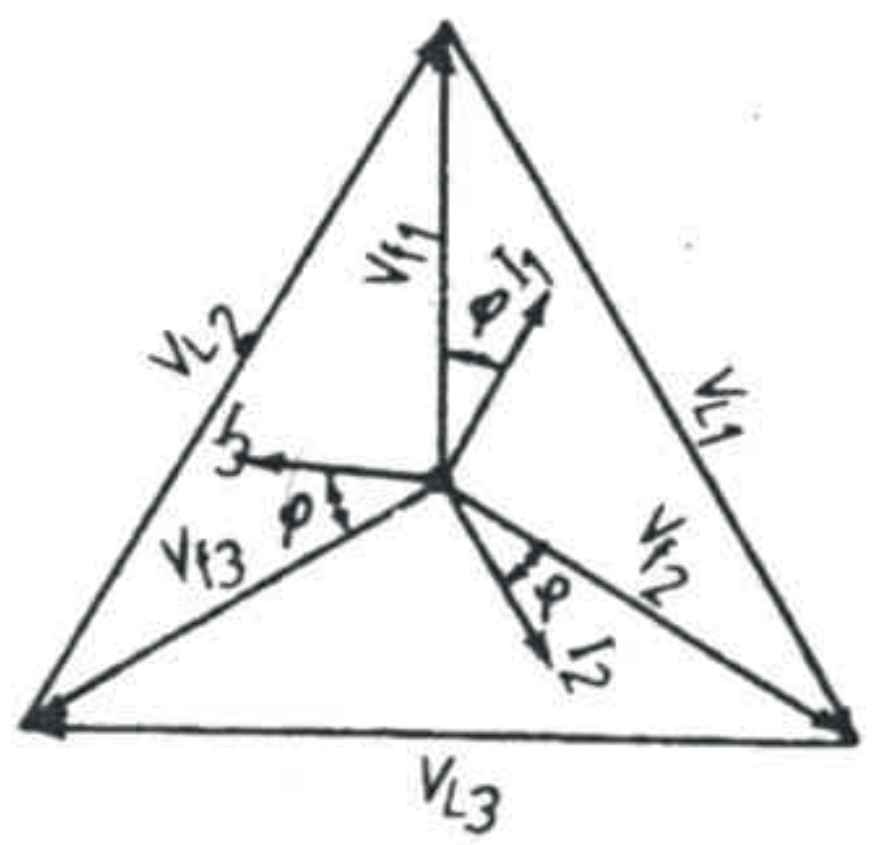


Entonces, lógicamente, la corriente irá retrasada un cierto ángulo j con respecto a la tensión. El ángulo de desfase se calculará por el procedimiento ya conocido de:

$\varphi=\operatorname{arctg} \frac{X}{R}$

El siguiente caso a analizar es la conexión en triángulo (D) de una carga equilibrada.

Por ello, supongamos ahora un sistema trifásico simétrico que alimenta una carga formada por tres impedancias iguales: $\mathrm{Z} 1=\mathrm{Z} 2=\mathrm{Z} 3=\mathrm{R}+\mathrm{j} \mathrm{X}$ conectadas en triángulo:

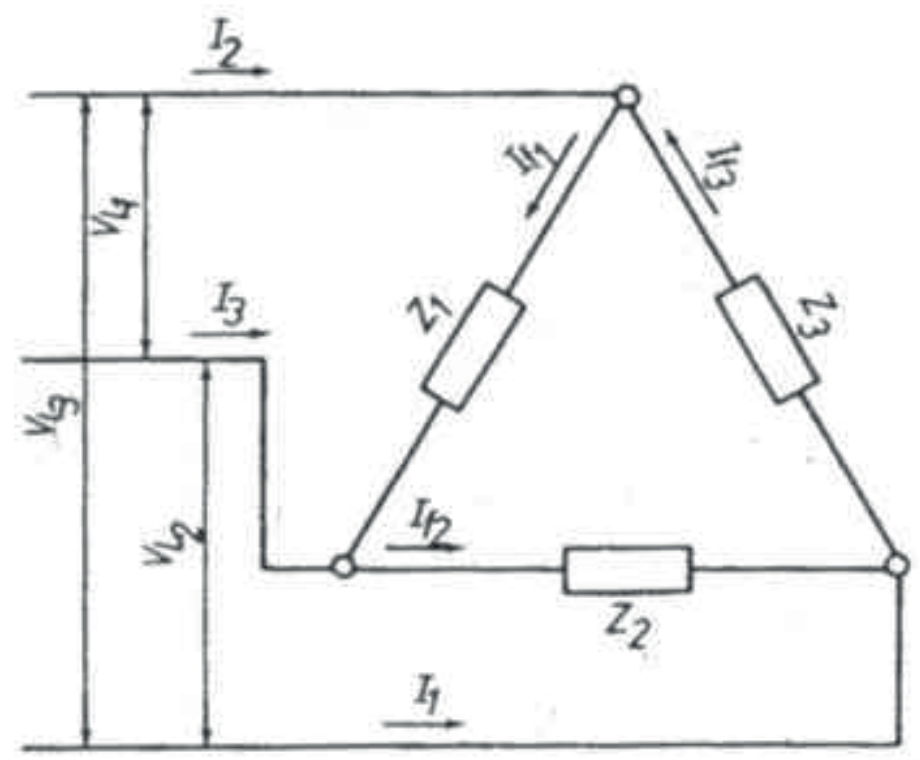

Las corrientes en cada una de las fases serán:

$I f_{1}=\frac{V l_{1}}{Z 1}=\frac{V l_{1}}{Z}$ 


$$
\begin{aligned}
& I f_{2}=\frac{V l_{2}}{Z 2}=\frac{V l_{2}}{Z} \\
& I f_{3}=\frac{V l_{3}}{Z 3}=\frac{V l_{3}}{Z}
\end{aligned}
$$

Sumando miembro a miembro las tres igualdades, resulta:

$$
I f_{1}+I f_{2}+I f_{3}=\frac{1}{Z}\left(V l_{1}+V l_{2}+V l_{3}\right)
$$

Recordando que:

$$
\eta l_{1}+7 l_{2}+\eta l_{3}=0
$$

Siendo necesariamente $\frac{1}{Z} \neq 0$, nos queda:

$$
I f_{1}+I f_{2}+I f_{3}=0
$$

Esta ecuación demuestra que, al tener una carga formada por tres impedancias iguales conectadas en triángulo, la suma vectorial de las corrientes de fase es nula. Este es el motivo por el que los vectores representativos de estas tres corrientes forman una estrella que arranca del baricentro del triángulo de las corrientes de línea: 


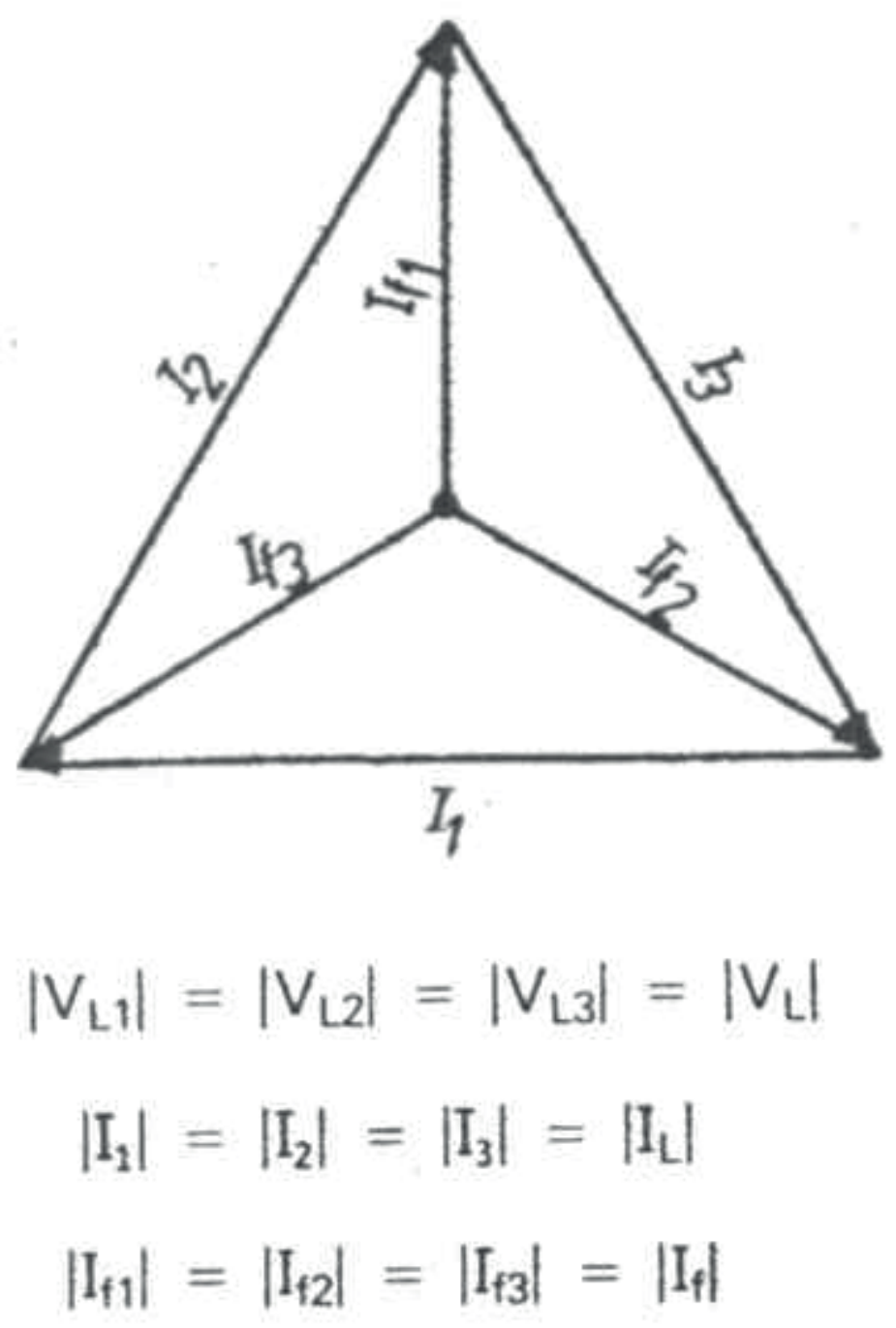

El cálculo de la corriente de línea, en función de la corriente de fase, cabe hacerlo con ayuda del triángulo: 


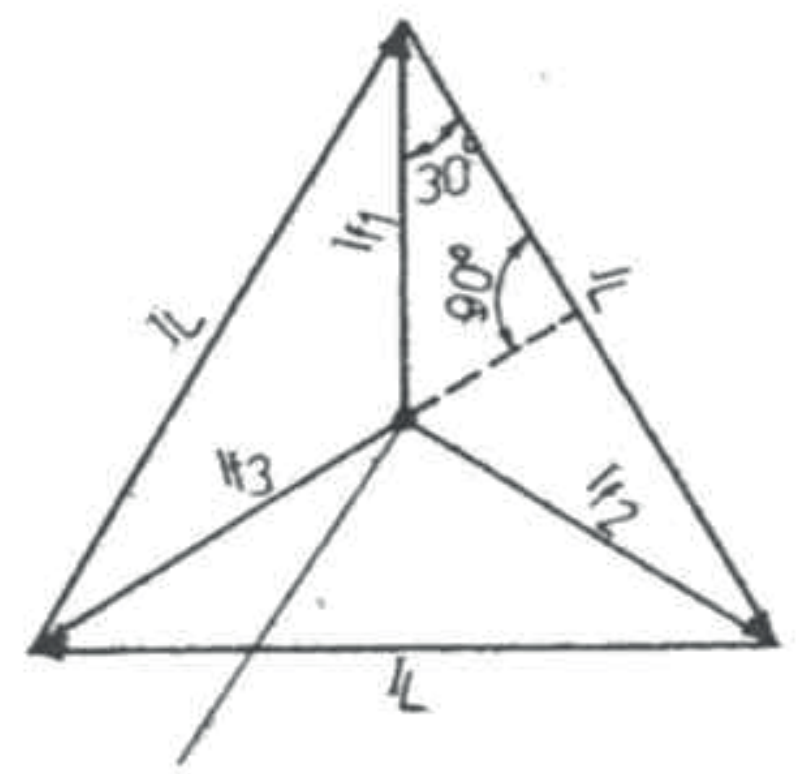

\section{baricentro}

En ella resulta que:

$$
\frac{I l}{2}=I f \cdot \cos 30^{\circ}
$$

Por tanto,

$$
I l=2 \cdot I f \cdot \cos 30^{\circ}=2 \cdot \text { If } \cdot \frac{\sqrt{3}}{2}=I f \cdot \sqrt{3}
$$

El diagrama vectorial de tensiones y corrientes podría ser el representado a continuación en el que se ha supuesto la carga $Z=R+j X$, óhmico-capacitiva: 


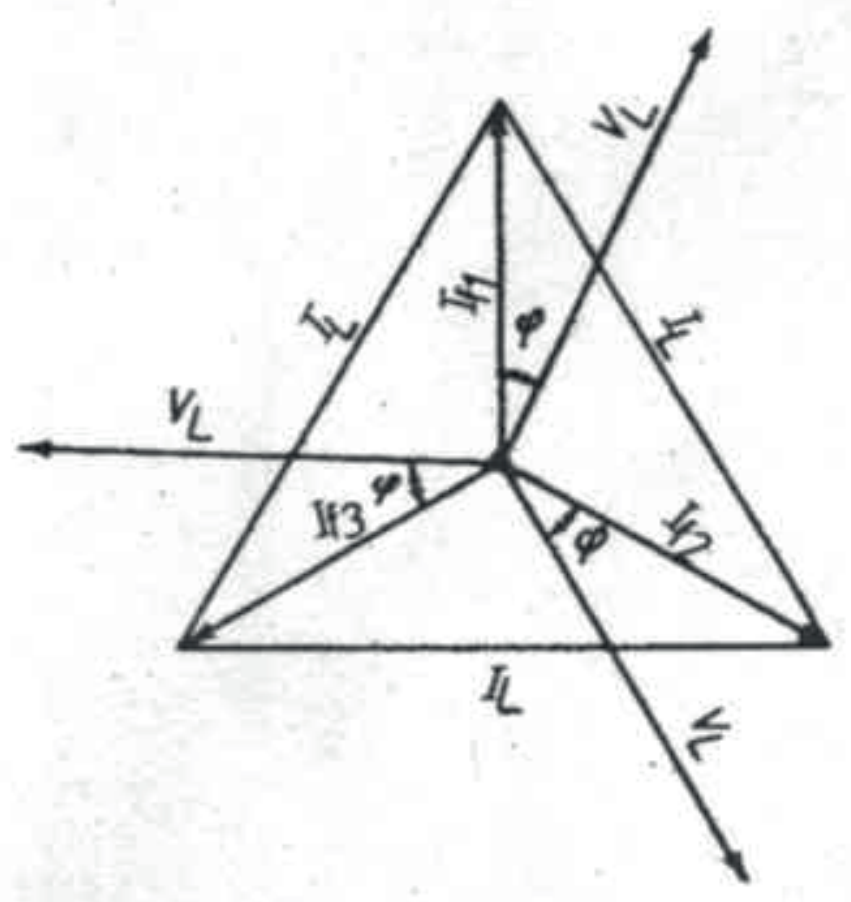

Evidentemente, la tensión irá retrasada en un cierto ángulo j con respecto a la corriente. Dicho ángulo de desfase se calculará por el procedimiento ya conocido:

$$
\varphi=\operatorname{arctg} \frac{X}{R}
$$

No siempre están equilibradas las cargas conectadas a las fases de los sistemas eléctricos; éste sería el caso de Z1 ${ }^{1}$ Z2 ${ }^{1}$ Z3. Esta condición nos obligará a calcular, por separado, las corrientes de fase o de línea, ya que en esta ocasión las corrientes son distintas y cada una tendrá un desfase respecto de la tensión, desfase que dependerá del tipo de carga. Los diagramas que se muestran a continuación nos muestran las representaciones de corrientes y tensiones de un circuito con cargas desequilibradas. 

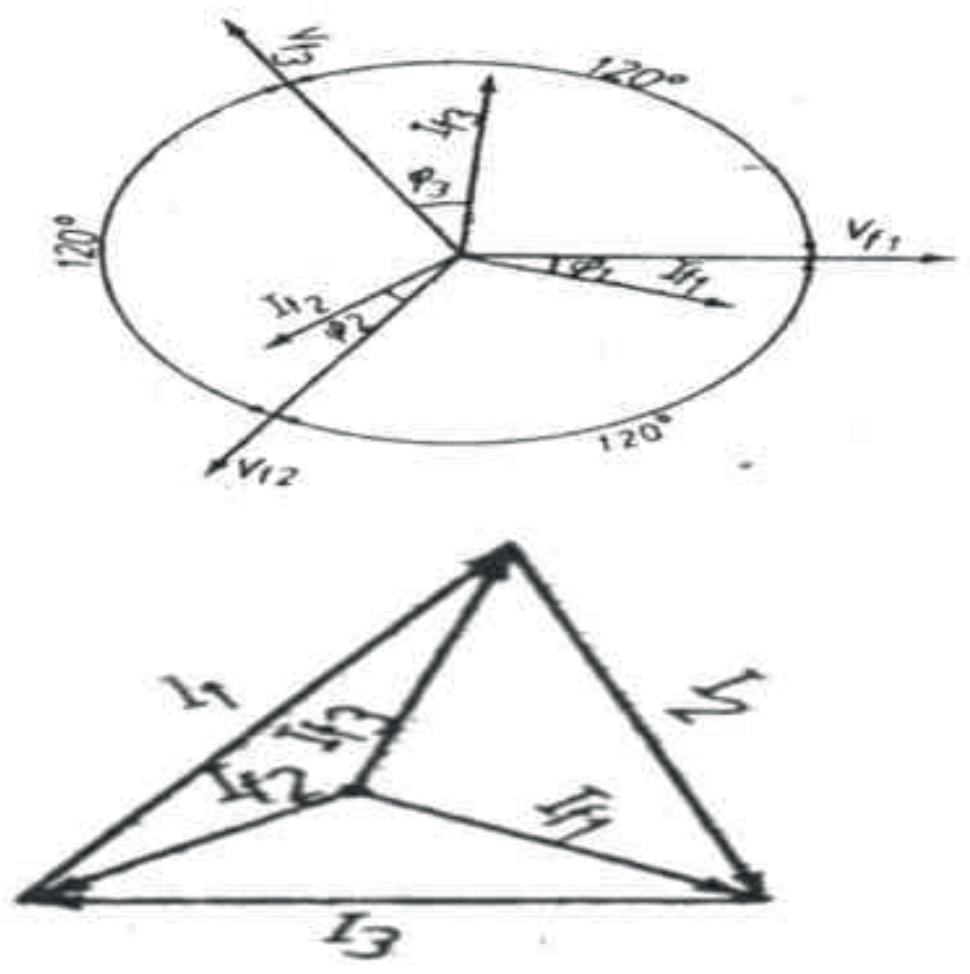

No profundizaremos más en el cálculo y proceso de este caso, por considerar que cada caso que nos encontráramos sería particular y se sale de las pretensiones de este pequeño estudio.

En resumen, la potencia activa de un sistema trifásico es igual a la suma aritmética de las potencias activas de las tres fases. La potencia reactiva total será igual a la suma algebraica de las potencias reactivas de las tres fases:

$$
\begin{aligned}
& P_{a t}=V_{11} \cdot I_{t 1} \cdot \cos \varphi_{1}+V_{12} \cdot I_{t 2} \cdot \cos \varphi_{2}+V_{t 3} \cdot I_{13} \cdot \cos \varphi_{3} \\
& P_{n}=V_{11} \cdot I_{11} \cdot \operatorname{sen} \varphi_{1}+V_{12} \cdot I_{2} \cdot \operatorname{sen} \varphi_{2}+V_{n} \cdot I_{2} \cdot \operatorname{sen} \varphi_{2}
\end{aligned}
$$

La potencia aparente total se calcula por el procedimiento ya conocido de la media cuadrática: 
$P t=\sqrt{P_{a t}^{2}+P_{r t}^{2}}$

Así la potencia activa total en los sistemas trifásicos equilibrados (recuérdese que en un sistema trifásico equilibrado, son iguales entre sí las tensiones de fase, las tensiones de línea, las corrientes de línea y las corrientes de fase) será tres veces la potencia activa de una sola fase:

$P_{a t}=3 \cdot V f \cdot$ If $\cdot \cos \varphi$

En la conexión estrella, puede ponerse:

$P_{a t}=3 \cdot \frac{V l}{\sqrt{3}} \cdot I l \cdot \cos \varphi=\sqrt{3} \cdot V l \cdot I l \cdot \cos \varphi$

Y en la conexión triángulo:

$P_{a t}=3 \cdot \frac{V l}{\sqrt{3}} \cdot I l \cdot \cos \varphi=\sqrt{3} \cdot V l \cdot I l \cdot \cos \varphi$

De lo que es fácil deducir que, en un sistema trifásico equilibrado, indistintamente conectado en estrella o en triángulo, la potencia activa total vale:

$P_{a t}=\sqrt{3} \cdot V l \cdot I l \cdot \cos \varphi$

Por análogos razonamientos pueden obtenerse los valores de la potencia reactiva y la aparente: 


\section{$P_{\mathrm{r}}=\sqrt{3} \cdot \mathrm{V}_{\mathrm{L}} \cdot \mathrm{I}_{\mathrm{L}} \cdot \operatorname{sen} \varphi$}

$$
P_{t}=\sqrt{3} \cdot V_{L} \cdot I_{L}
$$

En los sistemas desequilibrados es perfectamente válida la expresión general de las potencias activa, aparente y reactiva indicadas.

Hasta aquí este pequeño esquema centrado, fundamentalmente en los sistemas trifásicos de producción de tensión alterna. Si le ha quedado alguna duda, no tiene más que consultarla, escribiendo a la dirección electrónica de la revista. 